\title{
Phase diagram and quantum criticality of Heisenberg spin chains with Ising anisotropic interchain couplings
}

\author{
Yuchen Fan $\odot,{ }^{1}$ Jiahao Yang, ${ }^{2}$ Weiqiang $\mathrm{Yu},{ }^{1} \mathrm{Jianda} \mathrm{Wu} \odot,{ }^{2, *}$ and Rong $\mathrm{Yu}^{1, \dagger}$ \\ ${ }^{1}$ Department of Physics and Beijing Key Laboratory of Opto-Electronic Functional Materials and Micro-Nano Devices, \\ Renmin University of China, Beijing 100872, China \\ ${ }^{2}$ Tsung-Dao Lee Institute and School of Physics and Astronomy, Shanghai Jiao Tong University, Shanghai 200240, China
}

(Received 13 September 2019; revised manuscript received 14 February 2020; accepted 17 February 2020; published 19 March 2020)

\begin{abstract}
Motivated by recent progress on field-induced phase transitions in quasi-one-dimensional quantum antiferromagnets, we study the phase diagram of spin-1/2 Heisenberg chains with Ising anisotropic interchain couplings under a longitudinal magnetic field via large-scale quantum Monte Carlo simulations. The interchain interaction is shown to enhance longitudinal spin correlations and stabilize an incommensurate longitudinal spin density wave order at intermediate fields. With increasing field, the ground state changes to a canted antiferromagnetic order and the magnetization fully saturates above a quantum critical point, which is shown to fall into the $(3+2) \mathrm{D} X Y$ universality. In the quantum critical regime, the system experiences a fascinating dimensional crossover to a universal Tomonaga-Luttinger liquid (TLL) as demonstrated by the continuous change of the critical exponent of the specific heat. The TLL behavior extends to a broad field and temperature regime, as characterized by the calculated NMR relaxation rate $1 / T_{1}$. Our results not only explain a number of puzzling observations in a quasi-one-dimensional antiferromagnet $\mathrm{YbAlO}_{3}$ but also lay down a concrete ground to the study on these materials in general.
\end{abstract}

DOI: 10.1103/PhysRevResearch.2.013345

\section{INTRODUCTION}

In low-dimensional correlated electron systems, strong quantum fluctuations give rise to quantum phase transitions (QPTs) [1] and a number of exotic phenomena, such as unconventional superconductivity [2,3], non-Fermi-liquid behaviors [4,5], and quantum spin liquids [6]. In the past decade, tremendous progress has been made in understanding the nature of QPTs and associated emerging phenomena in quasi-one-dimensional (Q1D) spin systems. These include the quantum $E_{8}$ integrable system [7-9], many-body string excitations [10-12], novel quantun criticality $[13,14]$ in transverse field Ising chains, and Bose-Einstein condensation (BEC) and glassy phases in coupled antiferromagnetic (AFM) chains $[15,16]$.

As a paradigmatic model for 1D quantum antiferromagnets, the spin- $1 / 2$ Heisenberg chain is well described by a TLL, where both the longitudinal and transverse spin correlation functions follow algebraic decay [17]. Under a magnetic field, the staggered transverse correlations are always dominant over the longitudinal ones. As a result, a canted

\footnotetext{
*wujd@sjtu.edu.cn

†rong.yu@ruc.edu.cn
}

Published by the American Physical Society under the terms of the Creative Commons Attribution 4.0 International license. Further distribution of this work must maintain attribution to the author(s) and the published article's title, journal citation, and DOI.
AFM order with staggered transverse correlations (denoted as the TAF order) is stabilized when the interchain couplings turn on. For weakly coupled $X X Z$ spin chains with an Ising anisotropic intrachain exchange coupling, besides the TAF phase arising from a spin-flop mechanism [18], the peculiar quantum fluctuations give rise to incommensurate modulation of the longitudinal spin correlations [19], and can stabilize an incommensurate longitudinal spin density wave (LSDW) order [20,21]. This LSDW state has only recently been observed in $(\mathrm{Ba}, \mathrm{Sr}) \mathrm{Co}_{2} \mathrm{~V}_{2} \mathrm{O}_{8}$ within a narrow field range [12,22-26].

Recent inelastic neutron scattering (INS) measurements reveal quantum critical TLL behavior of a coupled spin-1/2 chain compound $\mathrm{YbAlO}_{3}$ with nearly isotropic (Heisenberg) intrachain exchange couplings [27]. Surprisingly, an incommensurate AFM state is observed over a broad field range. In this phase, the modulation of the ordering wave vector is proportional to the magnetization, which is a prominent characteristic of the LSDW order. The origin of this incommensurate AFM order leads to a major puzzle because within the framework of existing theory $[20,21]$, the LSDW state is only stabilized when the intrachain exchange coupling has an Ising anisotropy. The essence is the relevance of the interchain coupling, which was not considered in previous studies [20,22]. Though the importance of the interchain coupling for $\mathrm{YbAlO}_{3}$ was suggested recently [27,28], it is still generically an open question whether and how the interchain Ising anisotropy would affect the phase diagram and low-energy excitations of Heisenberg chains. This also poses a major challenge to the existing theories based on the interchain mean-field approximation [20], where the interchain fluctuations, crucial 
to the stabilization of the LSDW order in coupled Heisenberg chains, are neglected.

To tackle these issues, in this paper we study the fieldinduced phase diagram of spin-1/2 Heisenberg chains with Ising anisotropic interchain couplings by using large-scale quantum Monte Carlo (QMC) simulations. Our results unambiguously show that the interchain interactions enhance longitudinal spin correlations and further stabilize an incommensurate LSDW order. With increasing field, the ground state transforms from the LSDW to a TAF state, then to a fully polarized state by passing through a quantum critical point (QCP) controlled by the $(3+2) \mathrm{D} X Y$ universality. Increasing temperature above the QCP, the scaling of the thermal energy and the NMR relaxation rate demonstrate that the system undergoes a clear dimensional crossover to the universal TLL behavior, exhibiting rich physics and fine structure of the quantum criticality. We then propose NMR measurements as a means to probe the ground states and related low-energy excitations in Q1D antiferromagnets, including $\mathrm{YbAlO}_{3}$.

\section{MODEL AND METHOD}

We consider a model defined on a three-dimensional (3D) cuboidal lattice for the spin-1/2 Heisenberg chains with weak interchain couplings of the $X X Z$ type under a longitudinal magnetic field. The field is in parallel to the easy axis of spin, which is defined as the $z$ direction. The Hamiltonian reads as

$$
\begin{aligned}
H= & J_{c} \sum_{i} \vec{S}_{i} \cdot \vec{S}_{i+c}-g \mu_{B} H \sum_{i} S_{i}^{z} \\
& +J_{a b} \sum_{i, \delta=\{a, b\}}\left[\varepsilon\left(S_{i}^{x} S_{i+\delta}^{x}+S_{i}^{y} S_{i+\delta}^{y}\right)+S_{i}^{z} S_{i+\delta}^{z}\right] .
\end{aligned}
$$

Here $\vec{S}_{i}=\left\{S_{i}^{x}, S_{i}^{y}, S_{i}^{z}\right\}$ is a spin-1/2 operator defined at site $i$. $J_{c}$ and $J_{a b}$ are, respectively, the intrachain (along the $c$ axis) and interchain exchange couplings between the nearest neighbor spins. Note that in general $c$ and $z$ directions are not in parallel. $\varepsilon$ denotes the spin anisotropy of the interchain coupling, and only the Ising anisotropy $\varepsilon<1$ is considered in the current study. $g$ is the gyromagnetic factor, $\mu_{B}$ is the Bohr magneton, and $H$ is the applied magnetic field. We take $J_{c}$ as the energy unit and define the reduced temperature $t=T / J_{c}$ and reduced field $h=g \mu_{B} H / J_{c}$. Here we take $\varepsilon=0.25$ and $J_{a b}=0.2 J_{c}$ for demonstration. The effects of varying $\varepsilon$ and $J_{a b}$ on the phase diagram of the system will be discussed in Sec. VI. To study the model in Eq. (1), we perform numerically exact quantum Monte Carlo (QMC) simulations based on the stochastic series expansion (SSE) algorithm [29,30]. In the simulations, the largest system size is $32 \times 32 \times 256$ and the lowest temperature accessed is $t=0.003$.

\section{PHASE DIAGRAM AND THE LSDW PHASE}

Our main results are summarized in the phase diagram of Fig. 1(b), in which the thermal transition temperatures are determined from the peak position of the specific heat in Fig. 2. At low temperatures, three ordered phases appear successively with increasing field, and the respective spin patterns along a chain are illustrated in Fig. 1(a). An Ising AFM phase, with ordered moments aligned in the $z$ direc- (a)
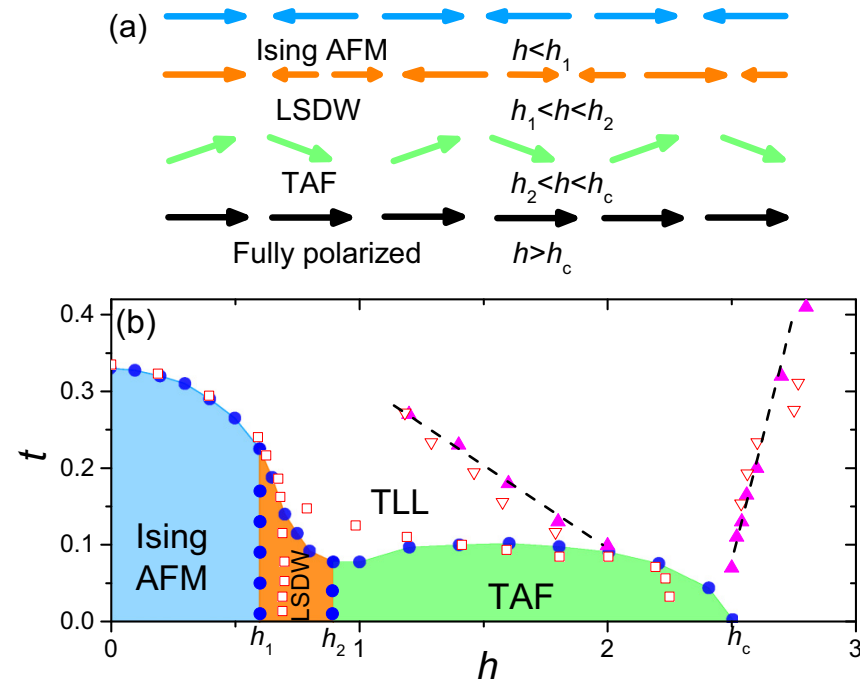

FIG. 1. (a) Sketch of the field evolution of ground-state spin patterns along one chain. (b) The thermal phase diagram of the model. Filled circles denote the phase boundaries, with the orderdisorder transitions determined by the specific heat data (see Fig. 2), and the transitions between ordered phases determined by the change of ordering wave vectors in the spin structure factors (see text). Also shown are the adapted experimental phase boundary data (open squares) for $\mathrm{YbAlO}_{3}$, from Ref. [27]. The filled and open triangles show the calculated and adapted experimental crossover temperatures close to the QCP at $h_{c}$, respectively. The dashed lines are linear fits.

tion, is stabilized for $h<h_{1}(\approx 0.6)$. An LSDW state with incommensurate longitudinal spin correlations is formed at an intermediate field regime $h_{1}<h<h_{2}(\approx 0.89)$. For $h>h_{2}$, the ground state becomes a TAF, which is a canted AFM state with staggered transverse magnetic components. Further increasing the field, the spins become fully polarized for $h>$ $h_{c}(\approx 2.50)$. The QPT at $h_{c}$ is continuous, while the transitions associated with the LSDW order at $h_{1}$ and $h_{2}$ are both first order.

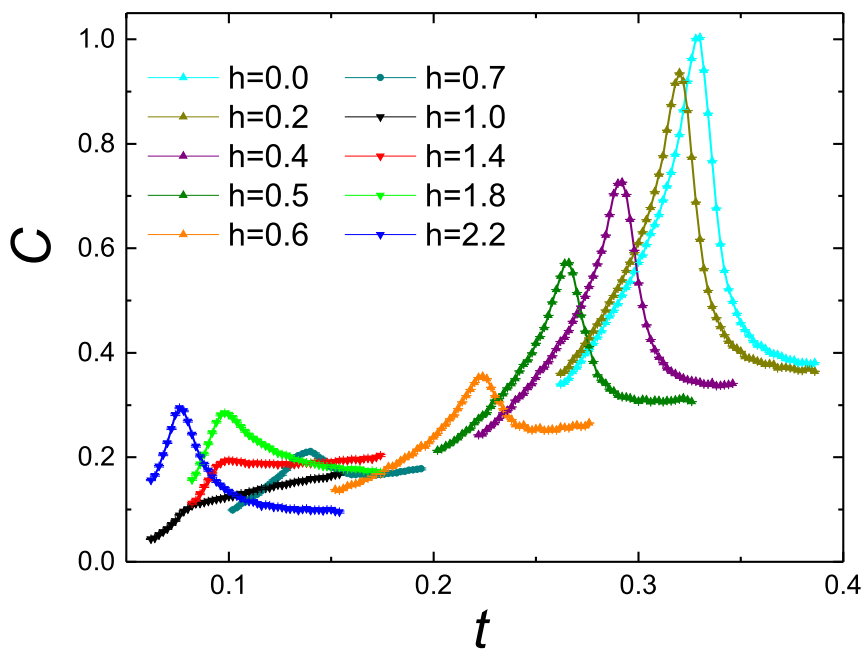

FIG. 2. Temperature dependence of specific heat $C$ at various field values, which is used to determine the phase boundary in Fig. 1. At each field, the transition to an AFM state is signaled as either a peak or a kinked downturn feature. 


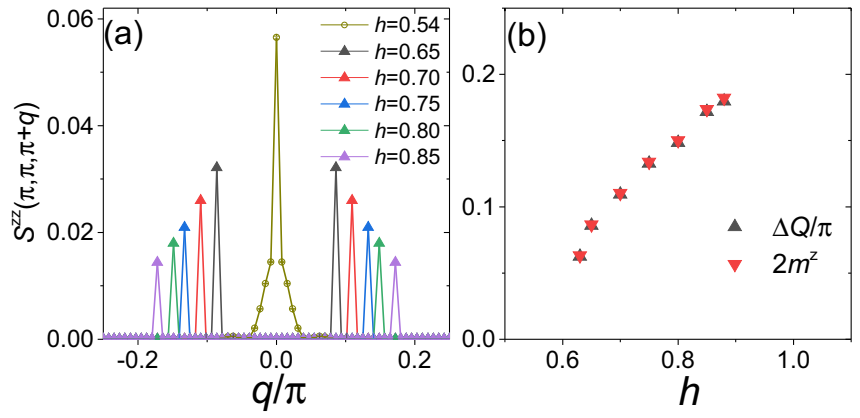

FIG. 3. (a) The longitudinal spin structure factor with wave number at various fields. The splitting of the peak signals the LSDW order. (b) Field evolutions of the shift of the ordering wave vector $\Delta Q$ and the magnetization $m^{z}$ (see text) in the LSDW phase.

To examine the nature of the ordered states, we calculate the normalized longitudinal and transverse spin structure factors

$$
\begin{aligned}
& \mathcal{S}^{z z}(\mathbf{q})=\frac{1}{N^{2}} \sum_{i j} e^{i \mathbf{q} \cdot\left(\mathbf{r}_{i}-\mathbf{r}_{j}\right)}\left\langle S_{i}^{z} S_{j}^{z}\right\rangle, \\
& \mathcal{S}^{x y}(\mathbf{q})=\frac{1}{2 N^{2}} \sum_{i j} e^{i \mathbf{q} \cdot\left(\mathbf{r}_{i}-\mathbf{r}_{j}\right)}\left\langle S_{i}^{x} S_{j}^{x}+S_{i}^{y} S_{j}^{y}\right\rangle .
\end{aligned}
$$

The Ising AFM order is signaled by a peak of $\mathcal{S}^{z z}(\mathbf{q})$ at $\mathbf{q}=(\pi, \pi, \pi)$. When $h>h_{1}$, we find that the peak splits into two, located at incommensurate wave vectors $\mathbf{q}=(\pi, \pi, \pi \pm$ $\Delta Q)$ [Fig. 3(a)]. The ordering wave vector shifts with increasing field, satisfying $|\Delta Q|=2 \pi m^{z}$ [Fig. 3(b)], a characteristic reflecting the Q1D TLL physics of the LSDW state [20]. This confirms that the incommensurate order is indeed a LSDW, which in this model arises from the enhancement of longitudinal correlations by interchain Ising anisotropy. For $h>h_{2}$, the peak of $\mathcal{S}^{z z}$ is suppressed, and the ground state changes to the TAF with a peak of $\mathcal{S}^{x y}(\mathbf{q})$ at $\mathbf{q}=(\pi, \pi, \pi)$, as shown in Fig. 4.

\section{QUANTUM CRITICALITY}

The QPT at $h_{c}$ takes place when the TAF order is suppressed. Since the TAF order breaks the spin $U(1)$ symmetry, the transition can be viewed as a field induced magnetic BEC [15,31] with a dynamical exponent $z=2$. The low-
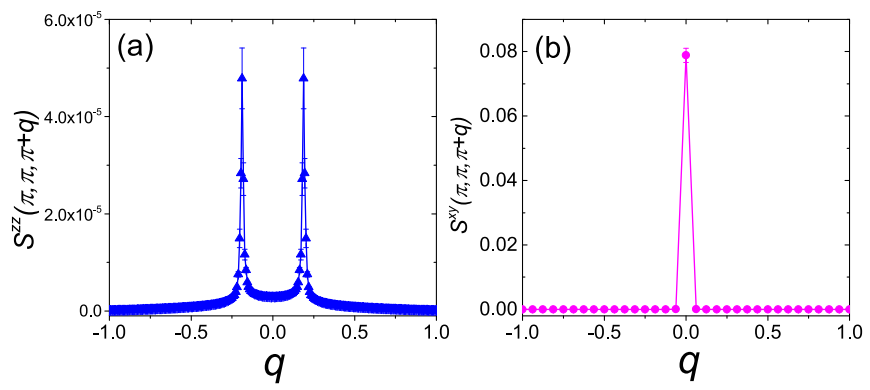

FIG. 4. $q$ dependence of the longitudinal (a) and transverse (b) spin structure factors, $\mathcal{S}^{z z}(\pi, \pi, \pi+q)$ and $\mathcal{S}^{x y}(\pi, \pi, \pi+q)$, respectively, at $t=0.05$ and $h=0.9$ in the TAF phase.
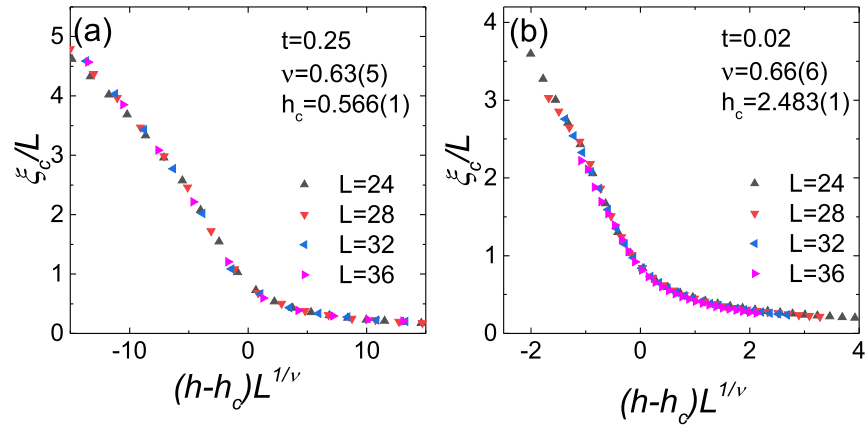

FIG. 5. Finite-size scaling of the correlation length along the $c$ axis, $\xi_{c}$, at thermal transitions to (a) the Ising AFM phase and (b) the TAF phase. The determined critical field $h_{c}(t)$ at the transition to TAF phase is plotted in Fig. 7(b), and the extracted correlation length exponent $v$ agrees with the value of 3D $X Y$ universality within the error bar.

energy effctive theory is then described by an $X Y$ model with an effective dimension $d+z$, where $d=3$ is the spatial dimension. Therefore, the QCP belongs to the $(3+2) \mathrm{D} X Y$ universality class. To show this explicitly, we first study the scaling behavior of the critical field $h_{c}(t)$ at low temperatures. We determine the critical field values independently from the scaling plot of correlation length $\xi \sim L$ (Fig. 5) and the peak of the field-dependent susceptibility $\chi^{z z}(h)=\partial m^{z} / \partial h$ [in Fig. 6(a)]. As shown in Fig. 7, the $h_{c}(t)$ data determined from either way follow the scaling relation of $3 d$ BEC, $h_{c}-h_{c}(t) \sim t^{d / 2}=t^{3 / 2}$. We then study the finite-temperature crossover in the vicinity of the QCP. At each field, the temperature-dependent susceptibility $\chi^{z z}(t)$ develops a broad peak, and the peak position defines the crossover temperature $T_{\text {cr }}$, as shown in Fig. 6(b). Near a QCP, $T_{\text {cr }} \sim\left|h-h_{c}\right|^{\nu z}$, where $v$ is the correlation length exponent. From Fig. 7(a), we find that $T_{\mathrm{cr}} \sim\left|h-h_{c}\right|$ on both sides of the QCP, consistent with the $(3+2) \mathrm{D} X Y$ universality $z=2$ and $v=1 / 2$.

Owing to its Q1D structure, the system is expected to display a $1 \mathrm{D}-3 \mathrm{D}$ crossover when decreasing the temperature. This is indeed shown in the scaling of thermal energy, $E=$ $\langle H\rangle / N$, right at the critical field $h_{c}$. Since $d E / d t=C \sim t^{d / z}$, where $C$ is the specific heat, one would expect $E \sim t^{\phi_{E}}$ with $\phi_{E}=d / z+1=5 / 2$. As shown in Fig. 8(a), this scaling fits only for $t \lesssim 0.06$. For $t \gtrsim 0.2, \phi_{E} \approx 3 / 2$, implying an
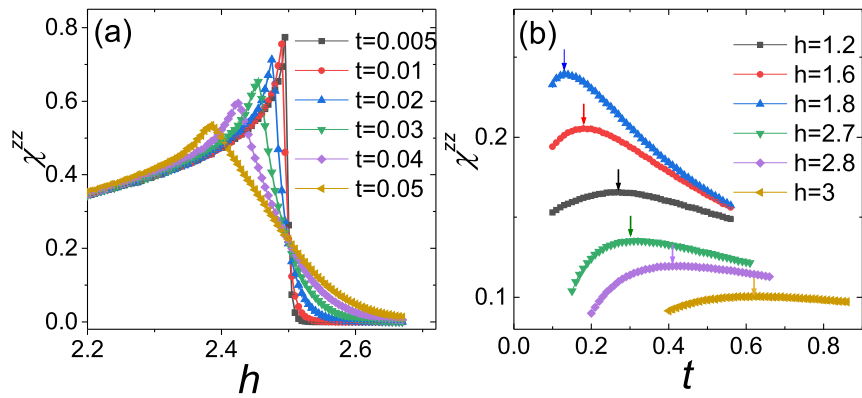

FIG. 6. (a) Field dependence of susceptibility $\chi^{z z}$ at low temperatures, where the peak position determines the critical field $h_{c}(t)$. (b) Temperature dependence of $\chi^{z z}$ above the ordering temperatures. The peak position (pointed by an arrow) determines the crossover temperature $T_{\text {cr }}$ in Figs. 1(b) and 7(a). 

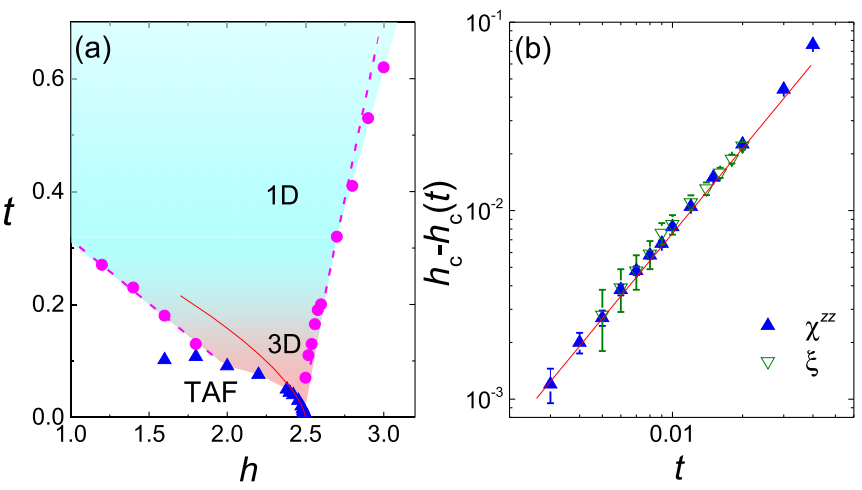

FIG. 7. (a) Critical fields $h_{c}(t)$ (blue triangles) and crossovers (red circles) near the QCP. The red solid line is a power-law fit $t \sim\left(h_{c}-h\right)^{2 / 3}$, and the dashed lines are linear fits. The color scheme illustrates a $1 \mathrm{D}-3 \mathrm{D}$ crossover in the quantum critical regime upon cooling. (b) Scaling of the critical fields $h_{c}(t)$ near the QCP determined from susceptibility and correlation length data. The line is a fit $h_{c}-h_{c}(t) \sim t^{3 / 2}$.

effective dimension $d_{\text {eff }}=1$. Careful windowing analysis [32] in Fig. 8(b) finds a gradual increase of $\phi_{E}$ from about $3 / 2$ to $5 / 2$ with $t$ decreasing from 0.2 to 0.06 , clearly indicating a $1 \mathrm{D}-3 \mathrm{D}$ crossover in this temperature regime. The crossover gives rise to peculiar quantum scaling behaviors. For example, the genuine 3D nature of the QCP is inherent in the lowtemperature scaling of the susceptibility data in the disordered phase [Fig. 8(d)], which satisfies

$$
\chi^{z z} \sim\left|h-h_{c}\right|^{\nu(d+z)-2} X\left(\frac{t}{\left|h-h_{c}\right|^{\nu z}}\right)
$$

with $d=3, v=1 / 2$, and $z=2$. In the quantum critical regime, it is expected that

$$
\chi^{z z} \sim\left|h-h_{c}\right|^{d / z+1-2 / v z} \tilde{X}\left(\frac{\left|h-h_{c}\right|}{t^{1 / v z}}\right)
$$
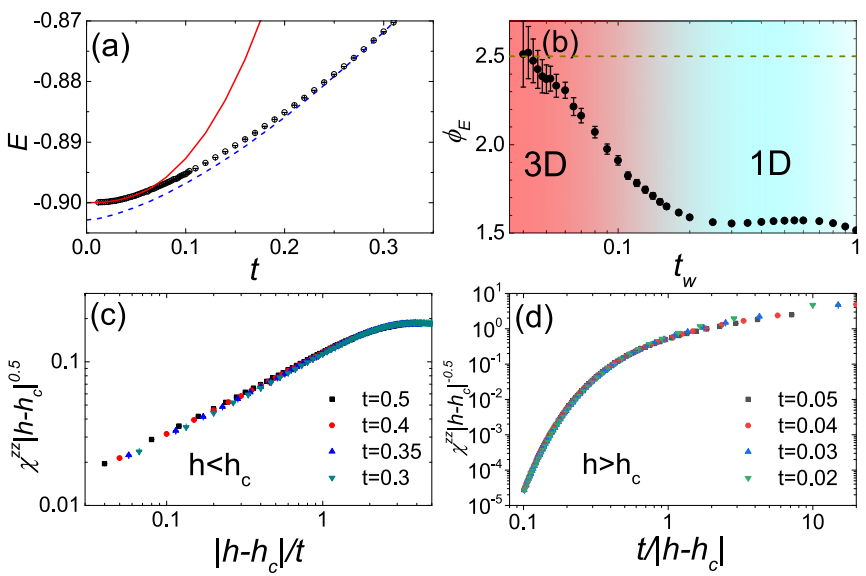

FIG. 8. (a) Temperature evolution of the thermal energy at $h_{c}$. The solid and dashed lines are power-law fits $E \sim t^{\phi_{E}}$ with $\phi_{E}=$ $5 / 2$ and $\phi_{E}=3 / 2$, respectively. (b) Windowing estimate on $\phi_{E}$ exhibiting a 1D-3D crossover. (c) Scaling of the high-temperature susceptibility data at $h<h_{c}$ showing an effective 1D quantum critical TLL behavior. (d) Scaling of the low-temperature susceptibility data at $h>h_{c}$ showing the $(3+2) \mathrm{D}$ nature of the QCP. with the same exponents at low temperatures, but the scaling of QMC data above the dimensional crossover temperature in Fig. 8(c) are consistent with $d_{\text {eff }}=1, v=1 / 2$, and $z=2$, characterizing a quantum critical TLL behavior.

\section{THE NMR RELAXATION RATE $1 / T_{1}$ AND THE TLL BEHAVIOR}

In the TLL regime of an $X X Z$ chain, the spin correlations decay algebraically as $\left\langle S_{0}^{z} S_{r}^{z}\right\rangle-\left(m^{z}\right)^{2} \sim \cos \left(2 k_{F} r\right) r^{-1 / \eta}$ and $\left\langle S_{0}^{x} S_{r}^{x}\right\rangle \sim(-1)^{r} r^{-\eta}$, where $k_{F}=\pi\left(1 / 2-m^{z}\right)$, denoting the Fermi wave number of pseudofermions mapped from the spin model by a Jordan-Wigner transformation, and the Luttinger exponent $\eta$ determines the decay rate. For a Heisenberg chain, $\eta<1$ for all fields, and the staggered transverse fluctuations always overwhelm the longitudinal ones. For an Ising anisotropic $X X Z$ chain, on the other hand, an $\eta$ inversion occurs at the field $h_{\text {inv }}$, e.g., the dominant fluctuation changes from longitudinal type with $\eta>1$ at $h<h_{\text {inv }}$ to transversetype with $\eta<1$ at $h>h_{\text {inv }}$ [20].

To examine whether the TLL behavior near $h_{c}$ extends to lower fields and to determine the dominant spin fluctuation associated with magnetic ordering, we calculate the NMR spin-lattice relaxation rate $1 / T_{1}$, which probes the low-energy spin fluctuations of a magnetic system [33,34]. For simplicity, the nuclear gyromagnetic ratio and the hyperfine coupling are set to be unity, and the longitudinal $(z z)$ and transverse $(x y)$ contribution to the relaxation rates are defined as $1 / T_{1}^{\alpha \alpha}=$ $\int d \mathbf{q} \operatorname{Im} \chi^{\alpha \alpha}\left(\mathbf{q}, \omega_{0}\right) / \beta \omega_{0}$, where $\beta=1 / t, \operatorname{Im} \chi^{\alpha \alpha}\left(\mathbf{q}, \omega_{0}\right)$ is the imaginary part of the dynamical susceptibility at the NMR frequency $\omega_{0} \rightarrow 0, \alpha=x, y, z$, and $1 / T_{1}^{x y}=1 / T_{1}^{x x}+1 / T_{1}^{y y}$. To avoid handling the analytical continuation in QMC simulations, we further adopt an approximation [35,36],

$$
1 / T_{1}^{\alpha \alpha} \approx \frac{2}{\pi t} \sum_{i}\left\langle\delta S_{i}^{\alpha}(\beta / 2) \delta S_{i}^{\alpha}(0)\right\rangle,
$$

where $\delta S_{i}^{\alpha}=S_{i}^{\alpha}-\left\langle S_{i}^{\alpha}\right\rangle$. Benchmark to this approximation is discussed in the Appendix, and the numerical results obtained from this approximation are quite reasonable.

$1 / T_{1}^{x y}$ and $1 / T_{1}^{z z}$ with temperatures for the $3 \mathrm{D}$ model are shown in Figs. 9(a) and 9(b). At $h=0.7$ where the ground state is a LSDW, the temperature-dependent $1 / T_{1}^{z z}$ develops a prominent peak at the ordering temperature, signaling enhanced critical fluctuations. In contrast, $1 / T_{1}^{x y}$ only shows a rapid downturn through the transition. At higher fields $h=1.0$ and 2.0, where the ground state is the TAF, the behavior reverses: A peak signaling the transition appears in $1 / T_{1}^{x y}$, whereas $1 / T_{1}^{z z}$ drops rapidly through the transition. Above the transition, we find an algebraic temperature dependence of $1 / T_{1}^{x y}$ [Fig. 9(c)], characterizing the TLL behavior. According to bosonization results, $1 / T_{1}^{x y} \sim T^{\eta-1}$ in a TLL [37]. Fitting to this function, we can extract the value of $\eta$ at each field. The $\eta$ with field is shown in Fig. 9(d). Surprisingly, $\eta>1$ for $h \lesssim 0.85$, indicating dominant longitudinal fluctuations despite the Heisenberg intrachain coupling. With increasing field, $\eta$ decreases monotonically, and across 1 at the inversion field $h_{\text {inv }} \approx 0.85$. Coincidentally, $h_{\text {inv }} \approx h_{2}$, which separates the LSDW and TAF ground states. The $\eta$ inversion and the peak feature in either $1 / T_{1}^{x y}$ or $1 / T_{1}^{z z}$ at the transition indicate 

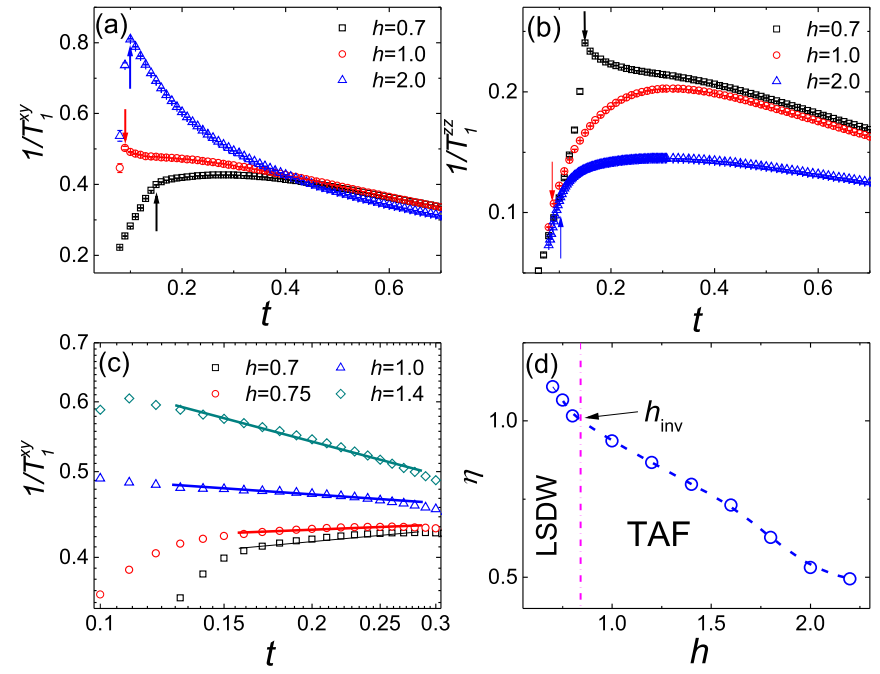

FIG. 9. (a) The transverse part of the NMR relaxation rate, $1 / T_{1}^{x y}$, with temperature at several fields. Arrows point to the ordering temperatures. (b) The longitudinal part $1 / T_{1}^{z z}$. (c) Same as panel (a) but in the double-logarithmic scale, showing the TLL behavior from the power-law fits (straight lines). (d) Extracted $\eta$ exponent from fits in panel (c). The dominant fluctuation changes from longitudinal type $(\eta>1)$ to transverse type $(\eta<1)$ across $h_{\text {inv }} \approx 0.85$. Here $h_{\text {inv }} \approx h_{2}$, which separates the LSDW and TAF orders [Fig. 1(b)].

that the condensation of the dominant fluctuations leads to the corresponding type of magnetic order.

\section{DISCUSSIONS AND CONCLUSION}

Our QMC results provide the first numerical evidence of a LSDW phase in a $3 \mathrm{D}$ model. The calculated $1 / T_{1}$ data unambiguously show that this phase is stabilized by the enhanced incommensurate longitudinal fluctuations. Such enhancement originates from the interchain Ising anisotropy. Note that this enhancement is not captured by the conventional mean-field theory [20], in which the Heisenberg intrachain couplings always lead to dominant transverse fluctuations because the dynamical effects of the interchain couplings are totally ignored.

At $h>h_{\text {inv }}$, the dominant transverse spin fluctuations not only account for the stabilization of the TAF order but also govern the quantum criticality, resulting in an 1D-3D crossover from the quantum critical TLL behavior at intermediate temperatures to the $(3+2) \mathrm{D} X Y$ universality at low temperatures as demonstrated. Such a scenario of quantum criticality generally holds for a broad class of weakly coupled $X X Z$ spin chain systems that have the same symmetry as the model in Eq. (1).

The phase diagram of the studied model is sensitive to the interchain coupling parameters $\varepsilon$ and $J_{a b}$. It is known that a large $J_{a b}$ favors a TAF order owing to the spin-flop mechanism [18]. For a fixed $J_{a b}$, the enhancement of longitudinal correlations, and hence the stabilization of LSDW, only take place when $\varepsilon$ is less than a critical value. As illustrated in Fig. 10, the LSDW is absent for $\varepsilon \gtrsim 0.5$ at $J_{a b} / J_{c}=0.2$. When $\varepsilon \rightarrow 0$, however, the transverse correlations survive
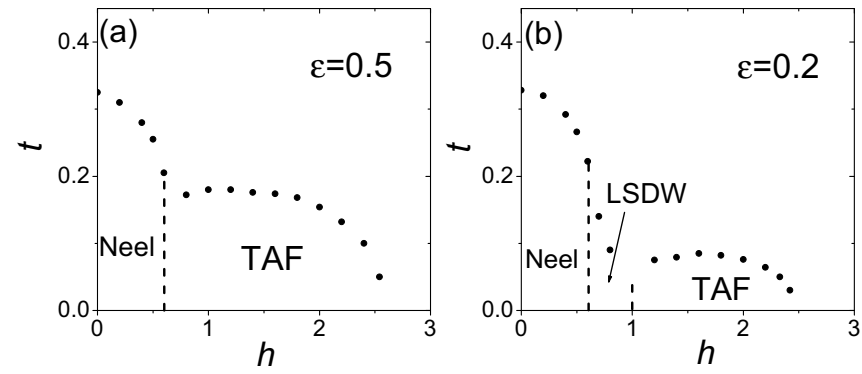

FIG. 10. Thermal phase diagram of the model in Eq. (1) of the main text for $J_{a b} / J_{c}=0.2$ and $\varepsilon=0.5$ [in panel (a)] and $\varepsilon=0.2$ [in panel (b)]. No LSDW phase is stabilized at $\varepsilon=0.5$. The TAF phase is stabilized in both cases.

only within each chain and hence cannot support a TAF order that breaks a continuous $U(1)$ symmetry. In this case, a QPT from the LSDW to the fully polarized phase with the $(3+1) D$ universality can be realized. For a finite $\varepsilon$, we always find a TAF phase before the magnetization is fully saturated (see Fig. 10), and hence the QPT is controlled by the $(3+2) \mathrm{D} X Y$ universality, irrelevant to the LSDW.

In what follows, we discuss the implication of our results for $\mathrm{YbAlO}_{3}$ and other related Q1D quantum magnets. It is found that the intrachain exchange coupling of $\mathrm{YbAlO}_{3}$ is almost isotropic, but the interchain one is dominated by the dipole-dipole interaction containing strong Ising anisotropy $[27,38]$. These are fully captured by our model in Eq. (1), where the interchain Ising anisotropy is ensured by the finite $\varepsilon<1$. Taking the measured values $J_{c} \sim 0.22 \mathrm{meV}$ and $g \sim$ 7.6 for $\mathrm{YbAlO}_{3}$ [27], we compare our results with experimental ones. As shown in Fig. 1(b), the phase boundary of the model agrees qualitatively with the adapted experimental one, though only an incommensurate AFM order is resolved in the measurement. The LSDW state in our model naturally explains the observed unusual incommensurate AFM order. Moreover, the linear scaling of $T_{\mathrm{cr}}$ and the quantum critical TLL behavior obtained in our theory have been observed in $\mathrm{YbAlO}_{3}$ [27]. The predicted genuine $(3+2) \mathrm{D}$ $X Y$ universality can be verified by future experiments in this compound and other coupled $X X Z$ spin chains, such as $(\mathrm{Ba}, \mathrm{Sr}) \mathrm{V}_{2} \mathrm{Co}_{2} \mathrm{O}_{8}$.

As suggested by INS measurements [27], the interchain exchange couplings in $\mathrm{YbAlO}_{3}$ contain both antiferromagnetic and ferromagnetic interactions. To avoid any complication in the theoretical treatment and for a clear discussion on the dominant physics, we take the interchain coupling $J_{a b}$ to be antiferromagnetic in the above model calculation, even though the consistency of the phase diagram with experiments indicate that our model has already captured the essential physics of the system. In fact, in our model the mechanism stabilizing the LSDW phase arises from the enhancement of the longitudinal spin correlations along the chain direction by Ising anisotropy of the interchain coupling, and this physics applies to systems with both ferromagnetic and antiferromagnetic interchain couplings. To see this more explicitly, we have carried additional calculations by taking $J_{a b}$ to be ferromagnetic. In Fig. 11, we show the calculated transverse and longitudinal structure factors at various fields with a 

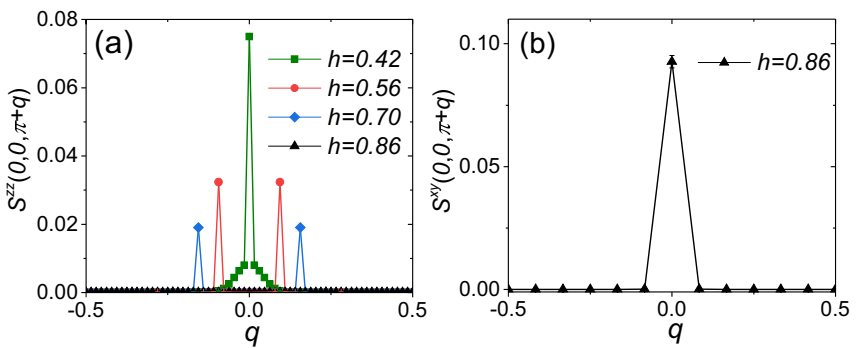

FIG. 11. $q$ dependence of the longitudinal (a) and transverse (b) spin structure factors at various fields with a ferromagnetic interchain coupling $J_{a b}=-0.2$ and $\varepsilon=0.25$. The splitting of the central peak in the longitudinal structure factor signals the LSDW order. For $h>h_{2} \approx 0.86$, the LSDW order is suppressed and the ground state has a TAF order.

ferromagnetic $J_{a b}=-0.2$ and $\varepsilon=0.25$. At $h>h_{1} \approx 0.5$ the peak of the longitudinal structure factor at $(0,0, \pi)$ splits, signaling an LSDW state. For $h>h_{2} \approx 0.85$, the LSDW order is suppressed and the ground state changes to the TAF order. Compared to the case with the antiferromagnetic interchain coupling, the LSDW and TAF orders are stabilized within similar field regimes. This result confirms that the key ingredient in understanding the experiment in $\mathrm{YbAlO}_{3}$ is the Ising anisotropy of the interchain coupling.

Our theory predicts a TAF order for $h>h_{2}$. In real materials, the LSDW and TAF orders may coexist or be phase separated [12,24]. In INS measurements, the possible phase separation and the extremely anisotropic gyromagnetic tensor in $\mathrm{YbAlO}_{3}$ make the transverse spin correlations hidden to neutron measurements. This certainly complicates the detection of the predicted TAF order and associated transverse spin fluctuations [27]. In light of the theoretical results, we hereby propose using NMR $1 / T_{1}$ to probe dominant low-energy spin fluctuations and associated magnetic ordering. Even when $1 / T_{1}^{z z}$ or $1 / T_{1}^{x y}$ each may be partially detectable through hyperfine coupling tensors, the evolution in the temperaturedependent $1 / T_{1}$ near the ordering temperature can still tell the dominant fluctuations and the orderings, as shown in Figs. 9(a) and 9(b). To further diagnose the dominant fluctuations and the underlying ground-state manifold, a careful examination of the possible $\eta$ inversion is called for. Given the universal property of the TLL, similar analysis can be applied to other related Q1D systems, such as $(\mathrm{Ba}, \mathrm{Sr}) \mathrm{V}_{2} \mathrm{Co}_{2} \mathrm{O}_{8}$, where the dominant fluctuations and the magnetic orders are still under debate $[12,24,25]$.

In conclusion, we study the field-induced phase diagram and quantum criticality of Q1D spin-1/2 AFM Heisenberg model with Ising anisotropic interchain couplings via largescale QMC simulations. We find that the interchain Ising anisotropy enhances incommensurate AFM correlations, stabilizing a LSDW ground state at low fields. The transverse spin correlations dominate at high fields and a TAF ground state is stabilized. The QCP is controlled by a $(3+2) \mathrm{D}$ $X Y$ universality and displays a clear 3D-1D crossover with increasing temperatures. The calculated NMR relaxation rates show enhanced critical fluctuations at the magnetic ordering, and the enhancement takes place at the particular channel relevant to the underlying magnetic order. Above the ordering temperature, the system exhibits universal TLL behavior and shows an $\eta$ inversion with increasing field, where the dominant spin fluctuation changes from the longitudinal type to the transverse one. These features make NMR an ideal low-energy probe for magnetic fluctuations and ordering in Q1D quantum magnets. Our findings thus shed light on future experimental and theoretical studies in these materials including $\mathrm{YbAlO}_{3}$.

\section{ACKNOWLEDGMENTS}

We acknowledge useful discussions with T. Giamarchi and L. S. Wu. This work was supported by the Ministry of Science and Technology of China (Grant No. 2016YFA0300504), the National Natural Science Foundation of China (Grants No. 11674392 and No. 51872328), the Fundamental Research Funds for the Central Universities, the Research Funds of Renmin University of China (Grant No. 18XNLG24), the Science and Technology Commission of Shanghai Municipality Grant No. 16DZ226020, and the Outstanding Innovative Talents Cultivation Funded Programs of Renmin University of China. R.Y. acknowledges hospitality at ENS de Lyon, France and at Tsung-Dao Lee Institute, Shanghai, China. J.W. acknowledges additional support from a Shanghai talent program.

\section{APPENDIX: BENCHMARK ON THE NMR RELAXATION RATE}

Here we benchmark the approximation in Eq. (6) by calculating the NMR relaxation rate $1 / T_{1}^{x y}$ of a Heisenberg chain. We compare the numerical results to analytical ones $[33,34]$ in Fig. 12. As one can see, the agreement between the numerical results and analytical ones is quite reasonable.

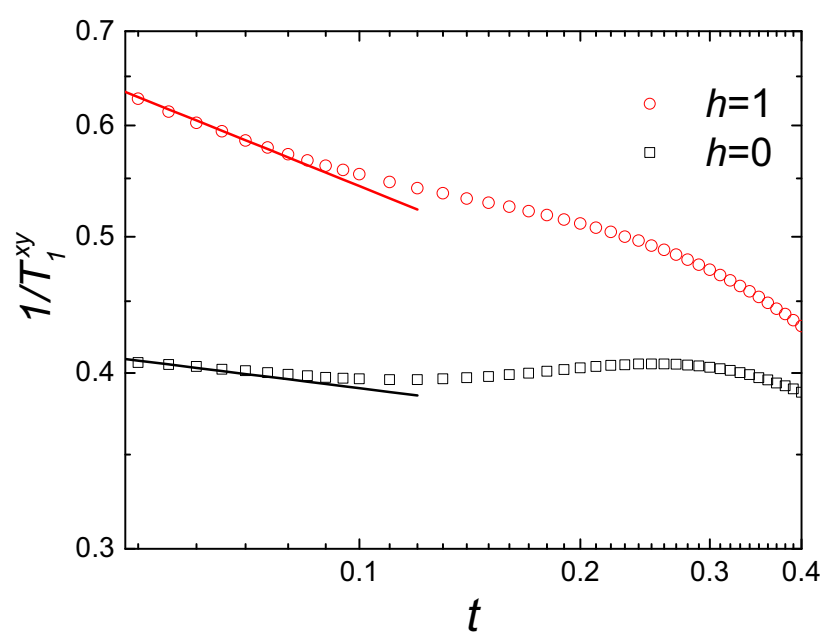

FIG. 12. Temperature dependence of the calculated transverse part of the NMR relaxation rate, $1 / T_{1}^{x y}$, in the double-logarithmic scale for a single Heisenberg chain at $h=0$ and $h=1$, respectively. The numerical data follow the predicted asymptotic behavior of TLL, as shown by the power-law fits (lines) with exponents given by analytical results in Refs. [33,34]. 
[1] S. Sachdev, Quantum Phase Transitions (Cambridge University Press, Cambridge, UK, 2011).

[2] P. A. Lee, N. Nagaosa, and X.-G. Wen, Rev. Mod. Phys. 78, 17 (2006).

[3] Y. Cao, V. Fatemi, S. Fang, K. Watanabe, T. Taniguchi, E. Kaxiras, and P. Jarillo-Herrero, Nature (London) 556, 43 (2018).

[4] G. R. Stewart, Rev. Mod. Phys. 73, 797 (2001).

[5] H. v. Löhneysen (ed.), Special issue on Quantum Phase Transitions, J. Low Temp. Phys. 161 (2010).

[6] J. Zheng, K. Ran, T. Li, J. Wang, P. Wang, B. Liu, Z. X. Liu, B. Normand, J. Wen, and W. Yu, Phys. Rev. Lett. 119, 227208 (2017).

[7] A. B. Zamolodchikov, Int. J. Mod. Phys. A. 04, 4235 (1989).

[8] R. Coldea, D. A. Tennant, E. M. Wheeler, E. Wawrzynska, D. Prabhakaran, M. Telling, K. Habicht, P. Smeibidl, and K. Kiefer, Science 327, 177 (2010).

[9] J. Wu, M. Kormos, and Q. Si, Phys. Rev. Lett. 113, 247201 (2014).

[10] Z. Wang, J. Wu, W. Yang, A. K. Bera, D. Kamenskyi, A. T. M. Nazmul Islam, S. Xu, J. M. Law, B. Lake, C. Wu, and A. Loidl, Nature (London) 554, 219 (2018).

[11] Z. Wang, M. Schmidt, A. Loidl, J. Wu, H. Zou, W. Yang, C. Dong, Y. Kohama, K. Kindo, D. I. Gorbunov et al., Phys. Rev. Lett. 123, 067202 (2019).

[12] A. K. Bera, J. Wu, W. Yang, Z. Wang, R. Bewley, M. Boehm, M. Bartkowiak, O. Prokhnenko, B. Klemke, A. T. M. Nazmul Islam, J. M. Law, and B. Lake, arXiv:1909.00146.

[13] Q. Faure, S. Takayoshi, S. Petit, V. Simonet, S. Raymond, L.-P. Regnault, M. Boehm, J. S. White, M. Månsson, C. Rüegg et al., Nat. Phys. 14, 716 (2018).

[14] Y. Cui, H. Zou, N. Xi, Zhangzhen He, Y. X. Yang, L. Shu, G. H. Zhang, Z. Hu, T. Chen, R. Yu, J. Wu, and W. Yu, Phys. Rev. Lett. 123, 067203 (2019).

[15] V. Zapf, M. Jaime, and C. D. Batista, Rev. Mod. Phys. 86, 563 (2014).

[16] R. Yu, L. Yin, N. S. Sullivan, J. S. Xia, C. Huan, A. PaduanFilho, N. F. Oliveira Jr, S. Haas, A. Steppke, C. F. Miclea et al., Nature (London) 489, 379 (2012).

[17] T. Giamarchi, Quantum Physics in One Dimension (Oxford University Press, Oxford, UK, 2004).

[18] M. E. Fisher and D. R. Nelson, Phys. Rev. Lett. 32, 1350 (1974).
[19] F. D. M. Haldane, Phys. Rev. Lett. 45, 1358 (1980).

[20] K. Okunishi and T. Suzuki, Phys. Rev. B 76, 224411 (2007).

[21] T. Suzuki, N. Kawashima, and K. Okunishi, J. Phys. Soc. Jpn. 76, 123707 (2007).

[22] S. Kimura, T. Takeuchi, K. Okunishi, M. Hagiwara, Z. He, K Kindo, T. Taniyama, and M. Itoh, Phys. Rev. Lett. 100, 057202 (2008).

[23] S. Kimura, M. Matsuda, T. Masuda, S. Hondo, K. Kaneko, N. Metoki, M. Hagiwara, T. Takeuchi, K. Okunishi, Z. He, K. Kindo, T. Taniyama, and M. Itoh, Phys. Rev. Lett. 101, 207201 (2008).

[24] B. Grenier, V. Simonet, B. Canals, P. Lejay, M. Klanjšek, M. Horvatić, and C. Berthier, Phys. Rev. B 92, 134416 (2015).

[25] M. Klanjšek, M. Horvatić, S. Krämer, S. Mukhopadhyay, H. Mayaffre, C. Berthier, E. Canévet, B. Grenier, P. Lejay, and E. Orignac, Phys. Rev. B 92, 060408(R) (2015).

[26] L. Shen, O. Zaharko, J. O. Birk, E. Jellyman, Z. He, and E. Blackburn, New J. Phys. 21, 073014 (2019).

[27] L. S. Wu, S. E. Nikitin, Z. Wang, W. Zhu, C. D. Batista, A. M. Tsvelik, A. M. Samarakoon, D. A. Tennant, M. Brando, L. Vasylechko et al., Nat. Commun. 10, 698 (2019).

[28] C. E. Agrapidis, J. van den Brink, and S. Nishimoto, Phys. Rev. B 99, 224423 (2019).

[29] O. F. Syljuåsen and A. W. Sandvik, Phys. Rev. E 66, 046701 (2002).

[30] F. Alet, S. Wessel, and M. Troyer, Phys. Rev. E 71, 036706 (2005).

[31] T. Giamarchi, C. Rüegg, and O. Tchernyshyov, Nat. Phys. 4, 198 (2008).

[32] S. E. Sebastian, P. A. Sharma, M. Jaime, N. Harrison, V. Correa, L. Balicas, N. Kawashima, C. D. Batista, and I. R. Fisher, Phys. Rev. B 72, 100404(R) (2005).

[33] E. Coira, P. Barmettler, T. Giamarchi, and C. Kollath, Phys. Rev. B 94, 144408 (2016).

[34] M. Dupont, S. Capponi, and N. Laflorencie, Phys. Rev. B 94, 144409 (2016).

[35] M. Randeria, N. Trivedi, A. Moreo, and R. T. Scalettar, Phys. Rev. Lett. 69, 2001 (1992).

[36] T. Roscilde (private communication).

[37] P. Bouillot, Ph.D. thesis, University of Geneva, Geneva, Switzerland, 2011.

[38] L. S. Wu, S. E. Nikitin, M. Brando, L. Vasylechko, G. Ehlers, M. Frontzek, A. T. Savici, G. Sala, A. D. Christianson, M. D. Lumsden, and A. Podlesnyak, Phys. Rev. B 99, 195117 (2019). 